\title{
Airway Mucosal Immune-suppression in Neonates of Mothers Receiving A(H1N1)pnd09 Vaccination During Pregnancy
}

Pedersen, Susanne Brix; Bischoff, Anne L.; Folsgaard, Nilofar V.; Vissing, Nadja H.; Birch, Sune; Bisgaard, Hans

Published in:

Pediatric Infectious Disease Journal

Link to article, DOI:

10.1097/INF.0000000000000529

Publication date:

2015

Document Version

Peer reviewed version

Link back to DTU Orbit

Citation (APA):

Pedersen, S. B., Bischoff, A. L., Folsgaard, N. V., Vissing, N. H., Birch, S., \& Bisgaard, H. (2015). Airway Mucosal Immune-suppression in Neonates of Mothers Receiving A(H1N1)pnd09 Vaccination During Pregnancy. Pediatric Infectious Disease Journal, 34(1), 84-90. https://doi.org/10.1097/INF.0000000000000529

\section{General rights}

Copyright and moral rights for the publications made accessible in the public portal are retained by the authors and/or other copyright owners and it is a condition of accessing publications that users recognise and abide by the legal requirements associated with these rights.

- Users may download and print one copy of any publication from the public portal for the purpose of private study or research.

- You may not further distribute the material or use it for any profit-making activity or commercial gain

- You may freely distribute the URL identifying the publication in the public portal 


\section{Airway Mucosal Immune-Suppression in Neonates of Mothers Receiving A(H1N1)pnd09 Vaccination During Pregnancy}

Anne Louise Bischoff $*^{1}$, MD, PhD; Nilofar V Følsgaard*1 ${ }^{1}$, MD, PhD; Nadja H Vissing ${ }^{1}$, MD; Sune Birch ${ }^{1}$, MSE; Susanne Brix ${ }^{2}$, MSci, PhD; Hans Bisgaard ${ }^{1}$, MD, DMSci

1. Copenhagen Prospective Studies on Asthma in Childhood; COPSAC, Health Sciences, University of Copenhagen, Copenhagen University Hospital, Gentofte, Ledreborg Allé 34, Gentofte 2820, Copenhagen, Denmark, www.copsac.com

2. Center for Biological Sequence Analysis, Department of Systems Biology, Technical University of Denmark, Søltofts Plads, Building 224, 2800 Lyngby, Denmark

Correspondence and request for reprints:

Professor Hans Bisgaard

Copenhagen Prospective Studies on Asthma in Childhood

Health Sciences, University of Copenhagen,

Copenhagen University Hospital; Gentofte

Ledreborg Allé 34

DK-2820 Gentofte

Copenhagen

Denmark

Tel: +453977 7360

Fax: +453977 7129

E-mail: bisgaard@copsac.com

Website: www.copsac.com

\section{Sources of support}

The nested vaccine study was funded by Novartis Vaccines and Diagnostics as unrestricted institutional grant.

COPSAC is funded by private and public research funds all listed on www.copsac.com. The Lundbeck Foundation; The Danish Strategic Research Council, Technology and Innovation; the Pharmacy Foundation of 1991; Augustinus Foundation; the Danish Medical Research Council and 
The Danish Pediatric Asthma Centre provided core support for COPSAC. The funding agencies did not have any role in study design, data collection and analysis, decision to publish, or preparation of the manuscript. No funding was received from National Institutes of Health (NIH), Wellcome Trust or Howard Hughes Medical Institute (HHMI.)

\section{Key words:}

Influenza Vaccines Influenza A(H1N1)pnd09 Pregnancy Cytokines Chemokines

Abbreviated title:

Airway Immune Modulation in Neonates of H1N1 Vaccinated Mothers

Running title:

High TGF- $\beta 1$ in Neonates of Vaccinated Mothers

This paper has a Supplemental Digital Content. 


\section{Structured Abstract}

Word count abstract: 248

\section{Background:}

It is recommended to vaccinate pregnant women against influenza. A possibly impact on the immune expression of the fetus has never been studied.

We aim to study the immune signature in the upper airways and the incidence of infections in neonates born to mothers receiving Influenza A(H1N1)pnd09 vaccination during pregnancy.

\section{Methods:}

156 women from the unselected Copenhagen Prospective Study on Asthma in Childhood (COPSAC 2010$)$ received Influenza A(H1N1)pnd09-vaccination during the 2009 pandemic. 51 mothers received the vaccine during pregnancy and 105 after pregnancy. 332 neonates of nonvaccinated mothers were included as secondary controls. Nasal mucosal lining fluid was sampled in 488 neonates and assessed for IL-12p70, IP-10, IFN- $\gamma$, TNF- $\alpha$, MIP-1 $\beta$, MCP-1, MCP-4, IL-4, IL5, IL-13, eotaxin-1, eotaxin-3, TARC , MDC, IL-17, IL-1 $\beta$, IL-8, TGF- $\beta 1$, IL-10 and IL-2. Infections were monitored the first year of life by daily diary cards and clinical controls. Results:

Neonates of mothers vaccinated during pregnancy had significant up-regulation of TGF- $\beta 1$ (ratio=1.52 [1.22-1.90], $\mathrm{p}=0.0002)$, and corresponding down regulation $(\mathrm{p}<0.05)$ of IL-12p70, IFN$\gamma$, IL-5, eotaxin-1, TARC, MDC, IL-8 in comparison to those vaccinated after pregnancy. The lagtime from vaccination during pregnancy to assessment of the immune signature showed significant and positive association to up-regulation of TGF- $\beta 1$ levels $(\mathrm{P}=0.0003)$ and significant negative association to other mediators. The incidence of infections in early infancy did not differ between the study groups.

\section{Conclusion:}

Influenza $\mathrm{A}(\mathrm{H} 1 \mathrm{~N} 1)$ pnd09 vaccination during pregnancy up-regulates TGF- $\beta 1$ and down-regulates key mediators of the protective immunity, but the rate of clinical infections was not detectably affected 


\section{Unstructured Abstract}

In this paper we aim to study the immune signature of the upper airways and the incidence of infections in neonates born to mothers receiving Influenza $A(H 1 N 1) p n d 09$ vaccination during pregnancy. We find that vaccination during pregnancy up-regulates TGF- $\beta 1$ and down-regulates key mediators of the protective immunity, we find no influence on clinical infections. 


\section{Introduction}

Influenza vaccination of pregnant women is generally recommended for the protection of both women and children, ${ }^{1}$ in particular during the recent Influenza A(H1N1)pnd09 (H1N1pnd09) pandemic in $2009 .^{2,3}$

The outcome of influenza infection during pregnancy is more severe during seasonal influenza epidemics, ${ }^{4-6}$ and even graver during influenza pandemics. ${ }^{7-9}$ Accordingly, the hospital admission and mortality rates were higher in pregnant women with influenza than in the general population during the 2009 pandemic, ${ }^{10-12}$ with increased rates of stillbirth, perinatal and neonatal mortality in infected pregnant women. ${ }^{13}$

Influenza is also a major cause of morbidity and hospitalization in infants younger than six months. ${ }^{14-16}$ In particular the H1N1pnd09 pandemic caused more severe disease in the neonates and young infants than in older children, all unprotected from passive immunity to this novel virus. ${ }^{17}$

No vaccination is approved for infants younger than six months. ${ }^{18,19}$ Therefore influenza vaccination during pregnancy has been proposed as a safe way to protect infants, ${ }^{1,5,20}$ presumably by cocooning ${ }^{21,22}$ and from antibodies transmitted from the mother. ${ }^{1,15,23}$ Yet the evidence is ambiguous as to the protective efficacy in the infants of vaccines given during pregnancy. ${ }^{24}$ It is well known that the maternal millieu during pregnancy affects the fetal immune competence. 25-27 While there is no published evidence on the possible effect of vaccination during pregnancy on the neonatal immune status, influenza vaccination is generally considered safe for the pregnant mother and the fetus.

Therefore, to examine the in vivo immune signature at the upper airways, we analyzed the cytokine and chemokine signature of the upper airway mucosa in neonates 4 weeks after birth $^{26,28,29}$ and monitored the incidence of infections in the first year of life in infants of mothers receiving H1N1pnd09-vaccination during pregnancy versus neonates of mothers not vaccinated during pregnancy. ${ }^{30}$ Furthermore we examined whether the time of vaccination during pregnancy was of importance to the impact of the vaccination on the cytokine and chemokine signature. 


\section{Methods}

\section{Design of the COPSAC 2010 Cohort Study}

The novel COPSAC 2010 cohort is an ongoing unselected prospective clinical study of 743 pregnant women and their 700 children. The pregnant women were recruited during gestational week 20-24 in $2009-2010 .^{26}$ Key exclusion criteria were; chronic endocrine-, nephro- or cardiac diseases. The neonates were enrolled at one week of age excluding infants with severe congenital abnormality. ${ }^{26}$ Information on confounders and baseline data were collected by structured clinical interviews at the parents' first visit to the COPSAC research clinic during gestational week 24 and 36, and again at one and four weeks after birth.

The families were seen at the COPSAC research clinic at age one week, one month, three months, six months and at six monthly intervals hereafter for close clinical follow-up.

Daily diary cards were used to monitor infections in the infants in the first year of life. Infections were categorized as common cold, pharyngitis, otitis, pneumonia, fever and gastro-intestinal infection. Parents were instructed how to fill in the diaries at study inclusion, and the diary cards were reviewed with the research doctor at each visit to clarify symptom definitions. Additionally, the research doctor interviewed the parents about other illness and any health care contacts outside the research clinic.

The database was double-checked against source data by and was subsequently locked. Data validity was assured by compliance with Good Clinical Practice guidelines and quality control procedures. An audit trail was done routinely.

All bio-sampling, clinical assessments and data collection were performed by a medical doctor with pediatric training and captured in a dedicated database on a novel SQS server.

\section{Design of the Nested Influenza Vaccination Study}

Women participating in the COPSAC 2010 cohort study were invited into a nested randomized trial of the safety and immunogenicity of a standard dose of $7.5 \mu \mathrm{g}$ of the monovalent influenza A/California/2009 (H1N1pnd09) surface-antigen MF59-adjuvanted vaccine (Focetria®, Novartis). Details of the study was recently reported. ${ }^{30}$

The study was initiated November 2009 early in the H1N1pnd09 pandemic, i.e. in the middle of the cohort recruitment period allowing comparison of the vaccine effects in pregnant and post-partum women. The recruitment was hampered by two factors; primarily the Danish Health Authorities 
were ambiguous in their recommendations for vaccination of pregnant women changing their recommendations during the pandemic. This ambivalence caused the women to doubt the necessity and safety of the offered vaccination. Secondly the clinical symptoms of the pandemic proved less serious than expected causing the women to reject the offer of receiving the vaccine. ${ }^{30}$

\section{Measurements of Airway Inflammatory Mediators in Upper Airway Mucosal Lining Fluid of Neonates}

Airway mucosal lining fluid was collected at four weeks of age with a pair of $3 \times 15 \mathrm{~mm}$ strips of filter paper (Accuwik Ultra, fibrous hydroxylated polyester sheets, cat no.SPR0730, Pall Life Sciences, UK) as previously described in detail. ${ }^{28}$

The mucosal lining fluid was analyzed for levels of Interleukin (IL)-12p70, CXCL10 (IP-10), Interferon-gamma (IFN- $\gamma$ ), Tumor necrosis factor-alpha (TNF- $\alpha$ ), CCL4 (MIP-1 $\beta$ ), CCL2(MCP-1), CCL13 (MCP-4), IL-4, IL-5, IL-13, CCL11 (eotaxin-1), CCL26 (eotaxin-3), CCL17 (TARC ), CCL22 (MDC), IL-17, IL-1 $\beta$, CXCL8 (IL-8), Transforming growth factor beta (TGF- $\beta 1$ ), IL-10 and IL-2 as previously described in detail. ${ }^{26,28}$

\section{Covariates}

Covariates were decided a priori based on our previous analyses showing association between the airway immune signature of the neonates and maternal atopic disease ${ }^{26}$, older siblings in the house and airway colonization with pathogenic bacteria. ${ }^{29}$ Furthermore we adjusted for vaccination month because there is a seasonal bias in the data collection since vaccination of participants peaked around the start of the pandemic in the winter of 2009.

\section{Statistical analyses}

All mediator levels were log-transformed prior to analysis. The associations between cytokines/chemokines levels in the two vaccine groups and the associations to the time of vaccination were tested using generalized linear models (GLM), for unadjusted as well as adjusted analysis. Results are reported as ratios between mean values of cytokines and chemokines, in the upper airway mucosal lining fluid in neonates of mothers vaccinated during pregnancy compared to the control group(vaccinated after pregnancy) with $95 \%$ confidence intervals in squared brackets. Baseline characteristics in the three groups were compared using Fisher's exact test, Wilcoxon Rank sum test and one-way analysis of variance (Anova).

The association between maternal vaccination and cumulative incidence of infections in the offspring throughout the first year of life was assessed by Quasi-Poisson regression estimating 
Incidence Risk Ratios (IRR), adjusting for season of birth, older siblings, and maternal atopic disease. In the secondary analysis we further adjusted for participation in the randomized study. $\mathrm{P}$-value $\leq 0.05$ is considered significant.

The data processing was conducted using SAS version 9.2 (SAS Institute, Cary, NC).

\section{Results}

Baseline Characteristics: Vaccines and Mucosal Lining Fluid

The study included 156 neonates of mothers participating in the randomized controlled trial of standard dose $(7.5 \mu \mathrm{g})$ of the H1N1pnd09, MF59-adjuvanted vaccine (Focetria ${ }^{\circledR}$, Novartis) 51 of the neonates were born to mothers vaccinated during pregnancy and 105 were born to mothers vaccinated after pregnancy (Figure E1 in SDC).

A secondary analysis compared children of mothers vaccinated during pregnancy with the children of 332 non-vaccinated mothers (Figure E1 in SDC).

The median time from vaccination of the pregnant women to sampling of mucosal lining fluid was 130 days (interquartile range, 95-146 days). The median age at mucosal lining fluid sampling in all neonates was 31 days (SD 5).

The 51 women vaccinated during pregnancy and their children did not differ from the 105 women vaccinated after pregnancy and their children or the 332 non-vaccinated and their children with respect to gender; older siblings; gestational age at birth; birth weight; Apgar score one min after birth; baseline antibody titers; mode of delivery; mother's age at birth; exposure to antibiotics, alcohol, and smoking during third trimester of pregnancy; paternal atopy; and household income(Table E1in SDC).

\section{Baseline Characteristics: Diary Cards} 152 out of 156 families (97\%) included in the randomized study returned their diary cards for the first year of life. For the secondary analysis, 284 out of 332 non-vaccinated families (86\%), returned their diary cards, yielding a total clinical follow up on 436 children (Figure E1in SDC). The mean number of infectious episodes pr. child was 8.2 (SD 4, range 0-24). Incidence of infections was 
significantly associated to older siblings (IRR=1.11 [1.01-1.21], $\mathrm{P}=0.03$ ) and birth season (IRR=0.88 [0.78-1.00], $\mathrm{P}=0.047$ for children born in the Autumn compared to Summer, no significant difference between other seasons), but was not associated to maternal atopic disease or participation in the randomized study (Table 3).

Mucosal Immune Profiles in Neonates of Mothers Vaccinated During versus After Pregnancy Neonates of mothers vaccinated during pregnancy showed a significant up-regulation of TGF- $\beta 1$ (ratio=1.52 [1.22-1.90], $\mathrm{P}=0.0002$ ) compared to neonates of mothers vaccinated after pregnancy. Additionally, we found a significant $(\mathrm{P} \leq 0.05)$ down-regulation of IL-12p70, IFN- $\gamma$, IL-5, eotaxin-1, TARC, MDC, IL-8, and a non-significant trend of down-regulation of IL-1 $\beta$ ( $\mathrm{P}=0.06)$ and IP10(P=0.10). Results are adjusted for season of birth, maternal atopy, older siblings, and airway colonization with pathogenic bacteria strains at sampling time. The adjustment induces greater effect size (estimates) and enhances the precision of the results. (Table 1 and Figure 1).

\section{Effect of Lag-Time from Vaccination to Sampling of Mucosal Lining Fluid}

The lag-time from vaccination to the assessment of the immune signature showed a significant and positive association to the up-regulation of TGF- $\beta 1$ levels $(\mathrm{P}=0.0003)$ (Figure 2$)$, and a significant $(\mathrm{P} \leq 0.05)$ and negative association with IP-10, IFN- $\gamma$, IL-5, Eotaxin-3, TARC, MDC, IL-8 and IL-10 in the mucosal lining fluid (Figure E2 and Table E2 in SDC).

Mucosal Immune Profiles in Neonates of Mothers Vaccinated During Pregnancy versus nonvaccinated Controls.

Neonates of vaccinated mothers showed up-regulated TGF- $\beta 1$ (ratio=1.32[1.11- 1.57], $\mathrm{P}=0.002$ ) compared to neonates of non-vaccinated controls, a significant down-regulation of IFN- $\gamma$, IL-5, TARC, MDC $(\mathrm{P} \leq 0.05)$ and a non-significant trend of down-regulation of IL-12p70 ( $\mathrm{P}=0.07)$, IL-4 $(\mathrm{P}=0.08)$ and $\mathrm{IL}-13(\mathrm{P}=0.07)$. Results are adjusted for participation in the randomized study, season of birth, maternal atopy, older siblings, and airway colonization with pathogenic bacteria strains at sampling time. (Table 2 and Figure 3).

Clinical Infections in Children of Mothers Vaccinated During Pregnancy Versus After Pregnancy and non-vaccinated Controls

There was no significant difference in the incidence of infections during the first year of life in children of mothers vaccinated during pregnancy versus children of mothers vaccinated after pregnancy $($ aIRR=1.10 [0.89-1.36], $\mathrm{P}=0.37)$. Also there was no significant difference in incidence of infections in children of mothers vaccinated during pregnancy and non-vaccinated controls 
$($ aIRR=0.99[0.84-1.18], $\mathrm{P}=0.95)($ Table 3).

\section{Discussion}

\section{Principle findings}

Vaccination with H1N1pnd09 vaccine during pregnancy induces alternations in the primary mucosal line of defense in the upper airways of newborn children. TGF- $\beta 1$ is up-regulated and other key-mediators linked to protective immunity are down-regulated suggesting a compromised local immune defense. This effect is enhanced the earlier in the pregnancy the woman receives vaccination.

We found no differences in the incidence of infections in the first year of life in the infants.

\section{Strengths and Limitations of the Study}

The H1N1pnd09 pandemic emerged in Denmark when the recruitment to our birth cohort was half completed. Therefore this "Experiment by Nature" allowed us to compare the effects of H1N1pnd09 vaccination in H1N1pnd09 -naïve women during pregnancy and post-partum.

Furthermore the design of the study enables us to assess the effect of the vaccine given at different time points during pregnancy allowing us to demonstrate that the immune suppression, as determined by TGF- $\beta 1$ levels, was significantly associated with the lag-time from vaccination to birth.

It is a strength of this study that we see strong and similar response between all tested groups. Children of mothers vaccinated during pregnancy had up-regulated TGF- $\beta 1$ compared to children of mothers receiving vaccine after pregnancy and children of non-vaccinated women. This was further confirmed by the significant association between the lag-time from vaccination in pregnancy to birth (sampling) and the TGF- $\beta 1$ signal. Hereby the same conclusions are similar in 3 different models.

It is a further strength of this study that it is nested in the well-established ongoing COPSAC birth cohort clinical research center. The setup of trained research doctors following standard operating procedures ensures consistency in the data material. The birth cohort study is designed as a clinical study with deep phenotyping of mother and child including comprehensive information's obtained at repeated visits to the research unit. This allowed an extensive comparison of the case and control 
cohorts of women together with detailed clinical follow-up of the children thereby increasing data validity, reducing the data variation and strengthening the effect estimates.

The in vivo sampling of mucosal lining fluid has become a well validated method for non-invasive assessment of cytokines and chemokines in the airways of children ${ }^{28,26,29,31}$ allowing us to measure undiluted samples directly from a target organ for respiratory disease, avoiding the dilemma of dilution accompanying nasal lavage models and intensified response found in challenge models. ${ }^{32,33}$ It is a limitation to our study that we do not know the exact agent the children were infected with during their infections recoded in the daily diaries. One could speculate that we would find fewer cases of influenza in the children of vaccinated mothers and thereby comparably more cases of other infections.

It is a limitation of this design that cases and controls did not receive the vaccinations at the same time of year. Most women were vaccinated before the pandemic reached Denmark by the end of 2009, but we continued vaccinating the pregnant women into early 2010 and therefore these (few) pregnant women in the study had a higher risk of contracting the natural infection. This we controlled for by the baseline IgG specific for H1N1pnd09, which did not differ between groups. Our findings cannot be uncritically extrapolated to other vaccines but must be limited to the H1N1pnd09 with MF59-adjuvanted vaccine we used. It is a limitation to the study that only 51 women received the H1N1pnd09 vaccine during pregnancy. This limits the power to detect clinical effects on immune deviation from vaccination in pregnancy.

\section{Interpretation}

The children born of mothers receiving vaccination during pregnancy had significantly up-regulated TGF- $\beta 1$ and down-regulation of Th1, Th2 and Th17 related mediators. TGF- $\beta 1$ has specific effects associated with down-regulation of the transcription factors T-bet and GATA- $3^{34}$, necessary for commitment of naïve CD4+ T-cells into Th1 and Th2 lineages, respectively. Moreover, both T-bet and GATA-3 activation are needed for maturation of cytotoxic NK cells, and NK functionality is thus also inhibited by TGF- $\beta 1^{34}$. Since Th1 and NK cells are both sources of IFN- $\gamma$, and Th2 of IL5 , IL-4 and IL-13, the lower levels of IFN- $\gamma$ and IL-5 in neonates of vaccinated mothers could possibly be mediated via TGF- $\beta 1$-induced suppression, but other mechanisms may also be in play.

The pattern we found is similar to the general pattern seen during pregnancy, where it serves to protect the fetus from rejection by the mother's immune response. ${ }^{35}$ Activation of the mother's 
adaptive immune reactions during pregnancy may in principle increase the risk of rejection of the fetus as reflected in the increased risk of abortion and preterm birth during Influenza infections.$^{9,13}$ Hence, it may be hypothesized that the boosted adaptive immune response in the vaccinated mothers is responsible for the down-regulation of the fetal immune system seen in our study. Down regulation of Th1 and Th17 response could be speculated to be counterproductive to clearance of infections but our study shows no difference in incidence of infections in the children. The study size limits the strength of this conclusion and these findings must be subject to further scrutiny in larger randomized control studies.

Earlier studies on the protection of neonates by antenatal vaccination show that vaccination of the mother during pregnancy protects the infants against respiratory infections. ${ }^{36-38}$ Some studies propose a protection against Influenza virus due to passively transmitted antibodies ${ }^{38}$ while other studies propose a protection against Influenza morbidity caused by cocooning ${ }^{36-38}$. Yet other studies show the opposite and advocate an increased risk of respiratory infections in children of mothers vaccinated during pregnancy ${ }^{24}$ the latter may be in line with our immunological findings.

A study of a population based cohort suggested that maternal influenza infection during pregnancy was associated with a twofold increased risk of infantile autism. ${ }^{39}$ Another study finds decreased neonatal levels of both Th-1 cytokines (i.e. IFN- $\gamma$ ) and Th-2 cytokines (i.e. IL-4, IL-10) in children later developing Autism Spectrum Disorders. ${ }^{40}$ Our results link these observations by showing that maternal influenza down-regulates the neonatal level of cytokines and chemokines.

\section{Conclusion}

This is the first study to assess airway immunology in neonates of mothers vaccinated during pregnancy, thereby presenting an unprecedented opportunity to explore the influence of antenatal vaccination on neonatal mucosal cytokine and chemokine immunology. Our findings suggest that H1N1pnd09 vaccination during pregnancy affects the mucosal immune competence of the unborn child and that this affect is dependent of time from birth of the vaccination.

We observe a 1.6-fold increase in TGF- $\beta 1$, along with decreased levels of the remaining immune mediators in upper airway of neonates from vaccinated mothers demonstrating that the fetal immune response is indeed affected by the maternal immune activation propagated by the vaccination protocol.

This is a notable finding, underscoring the necessity for research into vaccination programs in 
1

2

3

4

5

6

7

8

9

10

11

12

13

14

15

16

17

18

19

20

21

22

23

24

25

26

27

28

29

30

31

32

33

34

35

36

37

38

39

40

41

42

43

44

45

46

47

48

49

50

51

52

53

54

55

56

57

58

59

60

61

62

63

64

65

pregnant women and their influence on the neonatal immunity, not only focusing on neonatal immunity against the specific disease and the vaccine antigens, but the general immune status of the newborn child. 


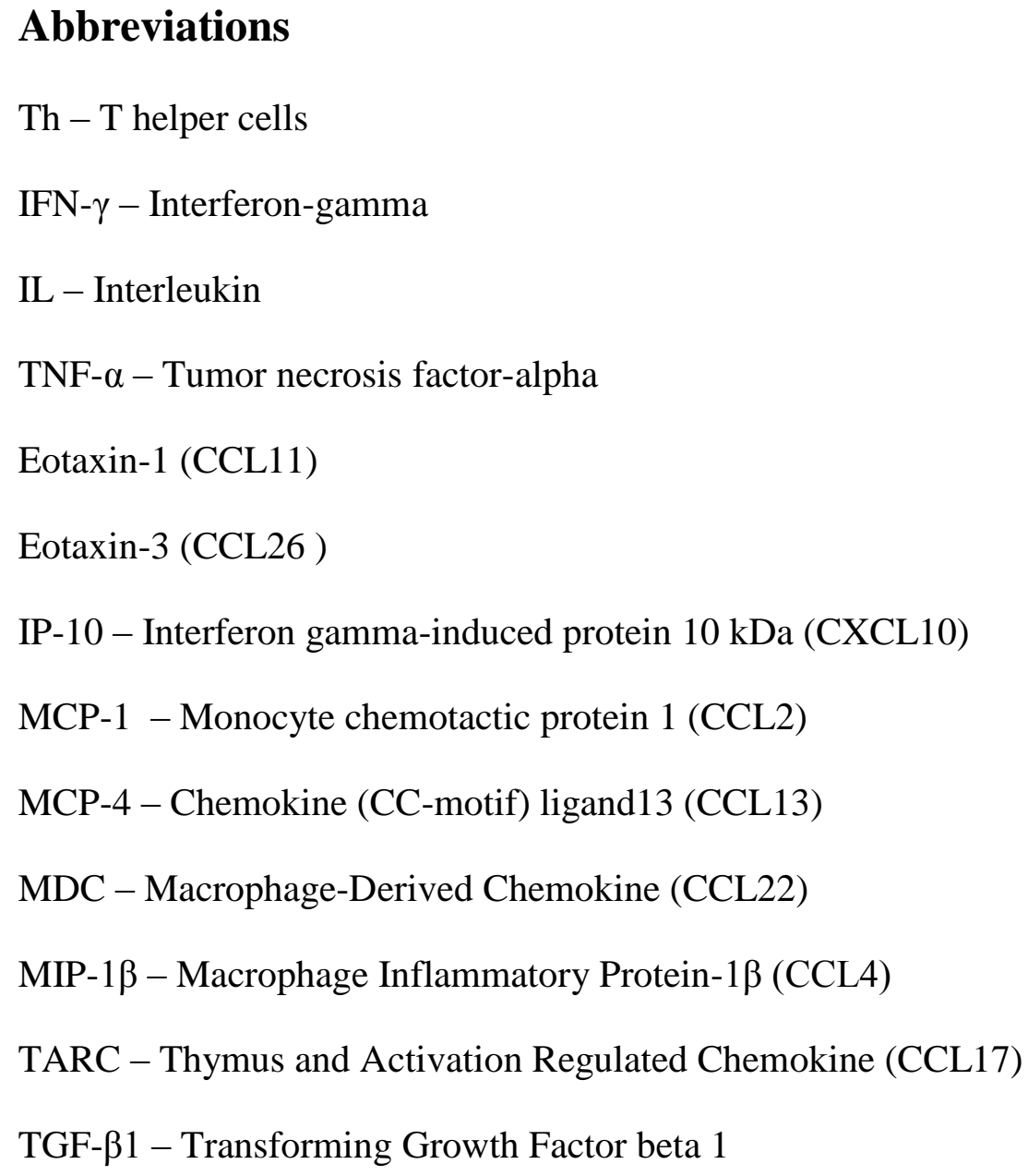




\section{Reference list}

1 Englund JA. Maternal immunization with inactivated influenza vaccine: rationale and experience. Vaccine 2003; 21: 3460-4.

2 Strategic Advisory Group of Experts on Immunization - report of the extraordinary meeting on the influenza A (H1N1) 2009 pandemic, 7 July 2009. Wkly Epidemiol Rec 2009; 84: 301-4.

3 Luteijn JM, Dolk H, Marnoch GJ. Differences in pandemic influenza vaccination policies for pregnant women in Europe. BMC Public Health 2011; 11: 819.

4 Neuzil KM, Reed GW, Mitchel EF, Simonsen L, Griffin MR. Impact of Influenza on Acute Cardiopulmonary Hospitalizations in Pregnant Women. American Journal of Epidemiology 1998; 148: $1094-1102$.

5 Blanchard-Rohner G, Siegrist C-A. Vaccination during pregnancy to protect infants against influenza: why and why not? Vaccine 2011; 29: 7542-50.

6 Skowronski DM, De Serres G. Is routine influenza immunization warranted in early pregnancy? Vaccine 2009; 27: 4754-70.

7 Elderfield R, Barclay W. Influenza Pandemics. In: Curtis N, Finn A, Pollard AJ, eds. Hot Topics in Infection and Immunity in Children VIII., Springer New York, 2011: 81-103.

8 Jamieson DJ, Theiler RN, Rasmussen SA. Emerging infections and pregnancy. Emerging Infect Dis 2006; 12: 1638-43.

9 Mosby LG, Rasmussen SA, Jamieson DJ. 2009 pandemic influenza A (H1N1) in pregnancy: a systematic review of the literature. American Journal of Obstetrics and Gynecology 2011; 205: $10-8$.

10 Critical illness due to $2009 \mathrm{~A} / \mathrm{H} 1 \mathrm{~N} 1$ influenza in pregnant and postpartum women: population based cohort study. BMJ 2010; 340: c1279.

11 Siston AM, Rasmussen SA, Honein MA, et al. Pandemic 2009 influenza A(H1N1) virus illness among pregnant women in the United States. JAMA 2010; 303: 1517-25.

12 Yates L, Pierce M, Stephens S, et al. Influenza A/H1N1v in pregnancy: an investigation of the characteristics and management of affected women and the relationship to pregnancy outcomes for mother and infant. Health Technol Assess 2010; 14: 109-82.

13 Pierce M, Kurinczuk JJ, Spark P, Brocklehurst P, Knight M, on behalf of UKOSS. Perinatal outcomes after maternal 2009/H1N1 infection: national cohort study. BMJ 2011; 342: d3214d3214.

14Libster R, Bugna J, Coviello S, et al. Pediatric hospitalizations associated with 2009 pandemic influenza A (H1N1) in Argentina. N Engl J Med 2010; 362: 45-55.

15 Munoz FM. Influenza virus infection in infancy and early childhood. Paediatric Respiratory Reviews 2003; 4: 99-104. 
16 Streng A, Grote V, Liese JG. Severe influenza cases in paediatric intensive care units in Germany during the pre-pandemic seasons 2005 to 2008. BMC Infectious Diseases 2011; 11: 233.

17 Bautista E, Chotpitayasunondh T, Gao Z, et al. Clinical aspects of pandemic 2009 influenza A (H1N1) virus infection. N Engl J Med 2010; 362: 1708-19.

18 Groothuis JR, Levin MJ, Rabalais GP, Meiklejohn G, Lauer BA. Immunization of high-risk infants younger than 18 months of age with split-product influenza vaccine. Pediatrics 1991; 87: 823-8.

19Piedra PA, Glezen WP, Mbawuike I, et al. Studies on reactogenicity and immunogenicity of attenuated bivalent cold recombinant influenza type A (CRA) and inactivated trivalent influenza virus (TI) vaccines in infants and young children. Vaccine 1993; 11: 718-24.

20Bednarczyk RA, Adjaye-Gbewonyo D, Omer SB. Safety of influenza immunization during pregnancy for the fetus and the neonate. American Journal of Obstetrics and Gynecology 2012; 207: S38-S46.

21 White PC, Baum DL, Ross H, Falletta L, Reed MD. Cocooning: Influenza Vaccine for Parents and Caregivers in an Urban, Pediatric Medical Home. CLIN PEDIATR 2010; 49: 1123-8.

22Emmanuel B. Walter M, Norma J. Allred P, Geeta K. Swamy M, Anne S. Hellkamp M, Rowena J. Dolor M. Influenza Vaccination of Household Contacts of Newborns: A Hospital-Based Strategy to Increase Vaccination Rates •. Infection Control and Hospital Epidemiology 2010; 31: 1070-3.

23 Naleway AL, Smith WJ, Mullooly JP. Delivering Influenza Vaccine to Pregnant Women. Epidemiologic Reviews 2006; 28: 47 -53.

24Black SB, Shinefield HR, France EK, Fireman BH, Platt ST, Shay D. Effectiveness of influenza vaccine during pregnancy in preventing hospitalizations and outpatient visits for respiratory illness in pregnant women and their infants. Am J Perinatol 2004; 21: 333-9.

25 Stensballe LG, Simonsen J, Jensen SM, Bønnelykke K, Bisgaard H. Use of Antibiotics during Pregnancy Increases the Risk of Asthma in Early Childhood. The Journal of Pediatrics 2012. doi:10.1016/j.jpeds.2012.09.049.

26Følsgaard NV, Chawes BL, Rasmussen MA, et al. Neonatal Cytokine Profile in the Airway Mucosal Lining Fluid Is Skewed by Maternal Atopy. Am J Respir Crit Care Med 2012; 185: 275 -280 .

27 Prescott SL. Early origins of allergic disease: a review of processes and influences during early immune development. Curr Opin Allergy Clin Immunol 2003; 3: 125-32.

28Chawes BLK, Edwards MJ, Shamji B, et al. A novel method for assessing unchallenged levels of mediators in nasal epithelial lining fluid. J Allergy Clin Immunol 2010; 125: 1387-1389.e3.

29Følsgaard NV, Schjørring S, Chawes BL, et al. Pathogenic Bacteria Colonizing the Airways in Asymptomatic Neonates Stimulates Topical Inflammatory Mediator Release. Am J Respir Crit 
Care Med 2013. doi:10.1164/rccm.201207-1297OC.

30Bischoff AL, Følsgaard NV, Carson CG, et al. Altered Response to A(H1N1)pnd09 Vaccination in Pregnant Women: A Single Blinded Randomized Controlled Trial. PLoS ONE 2013; 8: e56700.

31 Nicholson GC, Kariyawasam HH, Tan AJ, et al. The effects of an anti-IL-13 mAb on cytokine levels and nasal symptoms following nasal allergen challenge. J Allergy Clin Immunol 2011; 128: $800-807 . e 9$.

32Bisgaard H, Krogsgaard OW, Mygind N. Measurement of secretion in nasal lavage. Clin Sci 1987; 73: 217-22.

33 Pipkorn U, Proud D, Lichtenstein LM, Kagey-Sobotka A, Norman PS, Naclerio RM. Inhibition of mediator release in allergic rhinitis by pretreatment with topical glucocorticosteroids. $N$ Engl $J$ Med 1987; 316: 1506-10.

34 Marcoe JP, Lim JR, Schaubert KL, et al. TGF- $\beta$ is responsible for NK cell immaturity during ontogeny and increased susceptibility to infection during mouse infancy. Nature Immunology 2012; 13: 843-50.

35 Aluvihare VR, Kallikourdis M, Betz AG. Regulatory T cells mediate maternal tolerance to the fetus. Nat Immunol 2004; 5: 266-71.

36Benowitz I, Esposito DB, Gracey KD, Shapiro ED, Vázquez M. Influenza Vaccine Given to Pregnant Women Reduces Hospitalization Due to Influenza in Their Infants. Clin Infect Dis 2010; 51: 1355-61.

37Zaman K, Roy E, Arifeen SE, et al. Effectiveness of Maternal Influenza Immunization in Mothers and Infants. New England Journal of Medicine 2008; 359: 1555-64.

38Eick AA, Uyeki TM, Klimov A, et al. Maternal Influenza Vaccination and Effect on Influenza Virus Infection in Young Infants. Arch Pediatr Adolesc Med 2011; 165: 104-11.

39 Atladóttir HÓ, Henriksen TB, Schendel DE, Parner ET. Autism After Infection, Febrile Episodes, and Antibiotic Use During Pregnancy: An Exploratory Study. Pediatrics 2012. doi:10.1542/peds.2012-1107.

40 Abdallah MW, Larsen N, Mortensen EL, et al. Neonatal levels of cytokines and risk of autism spectrum disorders: An exploratory register-based historic birth cohort study utilizing the Danish Newborn Screening Biobank. J Neuroimmunol 2012; 252: 75-82. 


\section{Tables and Figures}

\section{Tables}

Table 1: Primary analysis of mediator levels in neonates born to mothers vaccinated during pregnancy versus mediator levels in neonates born to mothers vaccinated after pregnancy.

*Adjusted for season of birth, maternal atopy, older siblings, and bacterial colonization.

' False discovery rate $<0.05$ (Benjamini-Hochberg procedure for multiple testing correction)

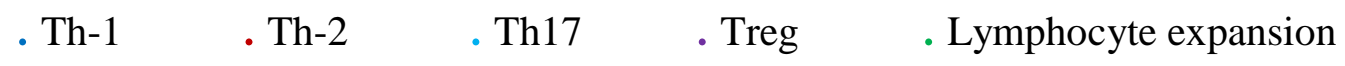

\begin{tabular}{|c|c|c|}
\hline Mediator & $\begin{array}{c}\text { Unadjusted } \\
\text { Ratio[CI-low, CI-high] P-value }\end{array}$ & \begin{tabular}{|c} 
Adjusted \\
* Ratio[CI-low, CI-high] P-value
\end{tabular} \\
\hline IL-12p70 & $0.74[0.52,1.04] P=0.08$ & $0.62[0.40,0.93] \quad \boldsymbol{P}=\mathbf{0 . 0 2}$ \\
\hline IP-10 & $0.92[0.54,1.58] \quad P=0.77$ & $0.58[0.30,1.11] P=0.10$ \\
\hline IFN- $\gamma$ & $0.62[0.40,0.97] \quad \boldsymbol{P}=\mathbf{0 . 0 4}$ & $0.33[0.19,0.57] \boldsymbol{P}=\mathbf{0 . 0 0 0 1}$, \\
\hline TNF- $\alpha$ & $1.04[0.60,1.80] P=0.88$ & $0.78[0.41,1.46] P=0.43$ \\
\hline MIP-1及 & $1.54[0.86,2.73] P=0.14$ & $0.95[0.50,1.82] P=0.88$ \\
\hline MCP-1 & $1.16[0.83,1.62] P=0.39$ & $0.83[0.54,1.27] P=0.39$ \\
\hline МCP-4 & $1.35[1.09,1.67] \quad P=0.006$ & $1.27[0.97,1.66] P=0.08$ \\
\hline IL-4 & $0.75[0.49,1.16] P=0.19$ & $0.79[0.47,1.34] \quad P=0.38$ \\
\hline IL-5 & $0.81[0.52,1.26] P=0.35$ & $0.49[0.30,0.82] \boldsymbol{P}=\mathbf{0 . 0 0 7 3}$, \\
\hline IL-13 & $0.72[0.51,1.00] P=0.05$ & $0.77[0.51,1.14] P=0.19$ \\
\hline Eotaxin-1 & $0.97[0.72,1.31] P=0.86$ & $0.66[0.45,0.97] \boldsymbol{P}=\mathbf{0 . 0 4}$ \\
\hline Eotaxin-3 & $0.99[0.68,1.46] \quad P=0.98$ & $0.72[0.44,1.18] P=0.19$ \\
\hline TARC & $0.76[0.60,0.97] \boldsymbol{P}=\mathbf{0 . 0 3}$ & $0.54[0.40,0.72] \boldsymbol{P}=7.0 \cdot 10^{-5}$, \\
\hline MDC & $0.63[0.46,0.85] \quad \boldsymbol{P}=\mathbf{0 . 0 0 3}$ & $0.38[0.26,0.54] \boldsymbol{P}=4.8 \cdot 10^{-7}$, \\
\hline IL-17 & $1.48[0.75,2.94] P=0.26$ & $1.29[0.54,3.10] P=0.56$ \\
\hline IL-1及 & $0.81[0.41,1.61] P=0.54$ & $0.48[0.22,1.04] P=0.06$ \\
\hline IL-8 & $0.94[0.59,1.52] P=0.81$ & $0.56[0.32,1.00] \boldsymbol{P}=\mathbf{0 . 0 5}$ \\
\hline TGF- $\beta 1$ & $1.38[1.16,1.64] \boldsymbol{P}=\mathbf{0 . 0 0 0 4}$ & $1.52[1.22,1.90] \boldsymbol{P}=\mathbf{0 . 0 0 0 2}$, \\
\hline IL-10 & $0.82[0.49,1.36] \quad P=0.44$ & $0.71[0.40,1.26] \quad P=0.24$ \\
\hline IL-2 & $1.09[0.71,1.67] P=0.69$ & $0.99[0.60,1.64] P=0.97$ \\
\hline
\end{tabular}


Table 2: Secondary analysis of mediator levels in neonates born to mothers vaccinated during pregnancy versus mediator levels in neonates of non-vaccinated controls.

*Adjusted for participation in the randomized study, season of birth, maternal atopy, older siblings, and bacterial colonization.

' False discovery rate $<0.05$ (Benjamini-Hochberg procedure for multiple testing correction)

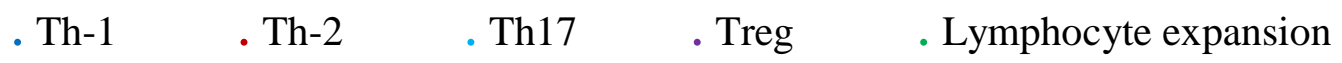

\begin{tabular}{|c|c|c|}
\hline Mediator & \begin{tabular}{|c} 
Unadjusted \\
Ratio[CI-low, CI-high] P-value
\end{tabular} & \begin{tabular}{|c|} 
Adjusted \\
$*$ Ratio[CI-low, CI-high] P-value
\end{tabular} \\
\hline IL-12p70 & $0.95[0.68,1.33] P=0.78$ & $0.71[0.48,1.03] P=0.07$ \\
\hline IP-10 & $1.27[0.82,1.96] \quad P=0.28$ & $0.88[0.52,1.48] P=0.63$ \\
\hline IFN- $\gamma$ & $1.13[0.73,1.73] P=0.59$ & $0.49[0.30,0.81] \boldsymbol{P}=\mathbf{0 . 0 0 5}$, \\
\hline TNF- $\alpha$ & $1.10[0.70,1.75] P=0.68$ & $0.93[0.55,1.58] P=0.80$ \\
\hline MIP-1及 & $1.24[0.76,2.01] P=0.39$ & $1.14[0.66,1.94] P=0.64$ \\
\hline MCP-1 & $1.16[0.87 .1 .55] P=0.31$ & $1.07[0.75 .1 .52] P=0.73$ \\
\hline MCP-4 & 1.24[1.01. 1.51] $P=0.04$ & $1.19[0.94 .1 .52] P=0.15$ \\
\hline IL-4 & $0.70[0.47,1.05] P=0.09$ & $0.65[0.40,1.05] P=0.08$ \\
\hline IL-5 & $0.80[0.54,1.18] P=0.26$ & $0.56[0.36,0.88] \quad \boldsymbol{P}=\mathbf{0 . 0 1}$ \\
\hline IL-13 & $0.82[0.60,1.12] P=0.22$ & $0.71[0.49,1.03] P=0.07$ \\
\hline Eotaxin-1 & $1.02[0.80,1.30] P=0.86$ & $0.90[0.67,1.21] P=0.48$ \\
\hline Eotaxin-3 & $1.34[0.93,1.95] P=0.12$ & $0.99[0.63,1.55] P=0.97$ \\
\hline TARC & $1.02[0.81,1.27] P=0.88$ & $0.72[0.55,0.94] \quad \boldsymbol{P}=\mathbf{0 . 0 2}$ \\
\hline MDC & $1.14[0.87,1.48] P=0.34$ & $0.60[0.44,0.82] \boldsymbol{P}=\mathbf{0 . 0 0 1}$, \\
\hline IL-17 & $1.34[0.77,2.35] \quad P=0.30$ & $1.22[0.63,2.38] P=0.55$ \\
\hline IL-1及 & $1.03[0.57,1.87] P=0.92$ & $0.63[0.32,1.23] \quad P=0.17$ \\
\hline IL-8 & $1.34[0.91,1.97] P=0.14$ & $0.81[0.51,1.28] P=0.36$ \\
\hline TGF- $\beta 1$ & $1.07[0.92,1.25] \quad P=0.35$ & $1.32[1.11,1.57] \boldsymbol{P}=\mathbf{0 . 0 0 2}$, \\
\hline IL-10 & $0.93[0.63,1.39] \quad P=0.73$ & $0.85[0.53,1.35] P=0.49$ \\
\hline IL-2 & $1.09[0.77,1.54] P=0.63$ & $1.10[0.73,1.65] P=0.65$ \\
\hline
\end{tabular}


Table 3: Risk of infections in offspring during the first year of life

\begin{tabular}{|c|c|c|}
\hline Risk of infections & crude IRR[CI-low, CI-high] P-value & aIRR[CI-low, CI-high] $P$-value \\
\hline $\begin{array}{l}\text { Vaccinated during pregnancy vs. after } \\
\text { birth }\end{array}$ & $0.98[0.83 ; 1.16] \quad P=0.81$ & $1.10[0.89 ; 1.36]^{*} \quad P=0.37$ \\
\hline $\begin{array}{l}\text { Vaccinated during pregnancy vs. non- } \\
\text { vaccinated controls }\end{array}$ & $1.00[0.86 ; 1.15] \quad P=0.95$ & $0.99[0.84 ; 1.18] \div \quad P=0.95$ \\
\hline$\$$ Adjusted for season of birth, maternal atopy, older siblings, and participation in the randomized study. & \multicolumn{2}{|c|}{ *Adjusted for season of birth, maternal atopy, and older siblings. } \\
\hline Confounding variables & crude IRR[CI-low, CI-high] $P$-value & \\
\hline Older siblings & $1.11[1.01 ; 1.21] \quad P=0.03$ & \\
\hline \multicolumn{3}{|l|}{ Birth season (ref=Summer) } \\
\hline Autumn & $0.88[0.78 ; 1.00] \quad \boldsymbol{P}=\mathbf{0 . 0 4 7}$ & \\
\hline Spring & $1.02[0.89 ; 1.17] \quad P=0.77$ & \\
\hline Winter & $0.99[0.88 ; 1.11] \quad P=0.81$ & \\
\hline Maternal Atopic Disease (yes/no) & $1.02[0.93 ; 1.11] \quad P=0.70$ & \\
\hline Participation in RCT & $1.04[0.92 ; 1.12] \quad P=0.75$ & \\
\hline
\end{tabular}


Figure 1: Ratios [95\% confidence interval] between mean values of cytokine and chemokine, in the upper airway mucosal lining fluid in healthy neonates of mothers vaccinated during pregnancy versus neonates born to mothers vaccinated after pregnancy. Data are adjusted for season of birth, maternal atopy, older siblings, and bacterial colonization.

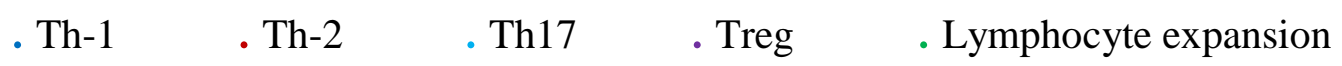

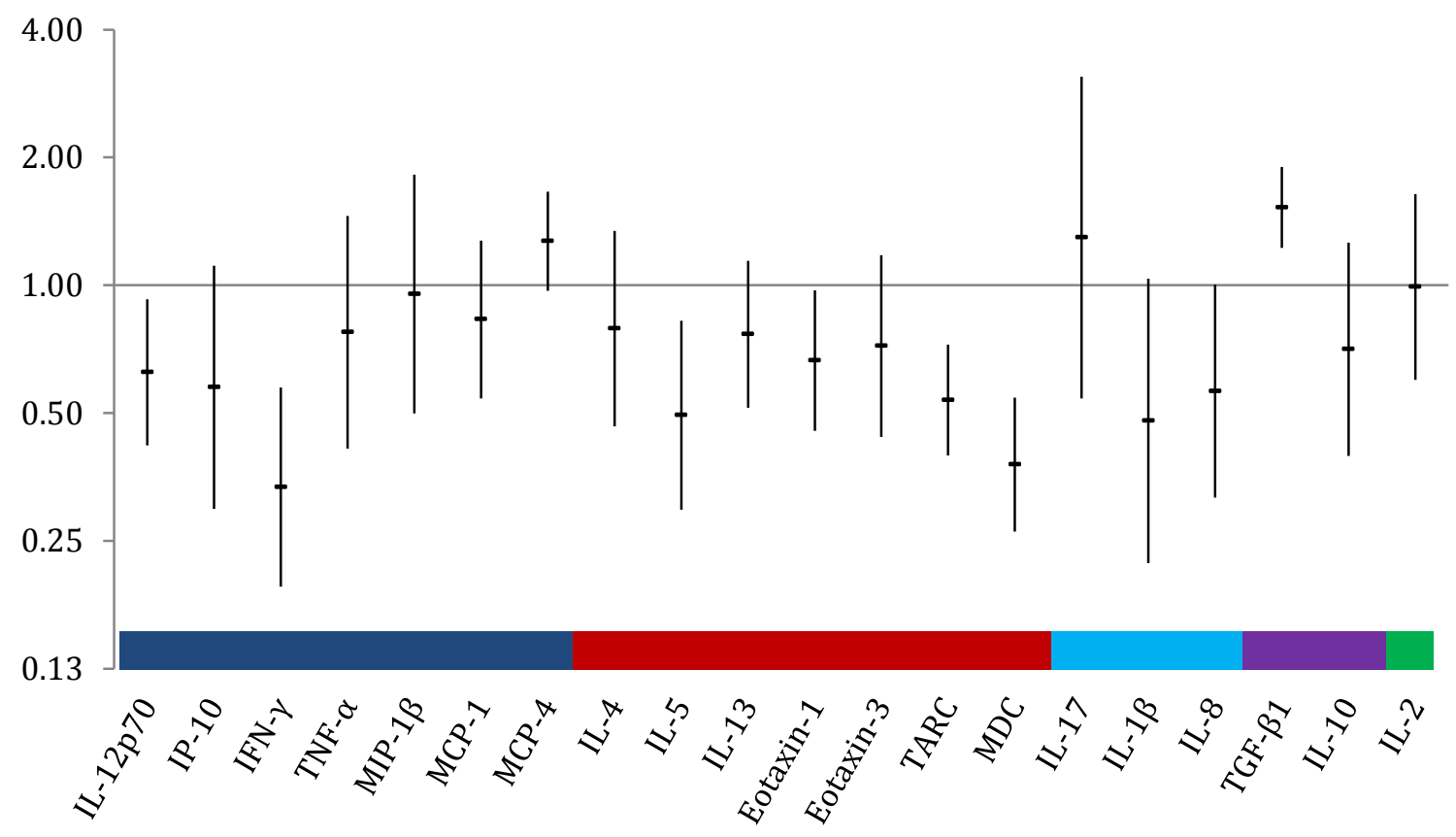


Figure 2: TGF- $\beta 1$ concentration vs. days from H1N1pnd09-vaccination during pregnancy to sampling of mucosal lining fluid from the neonatal offspring.

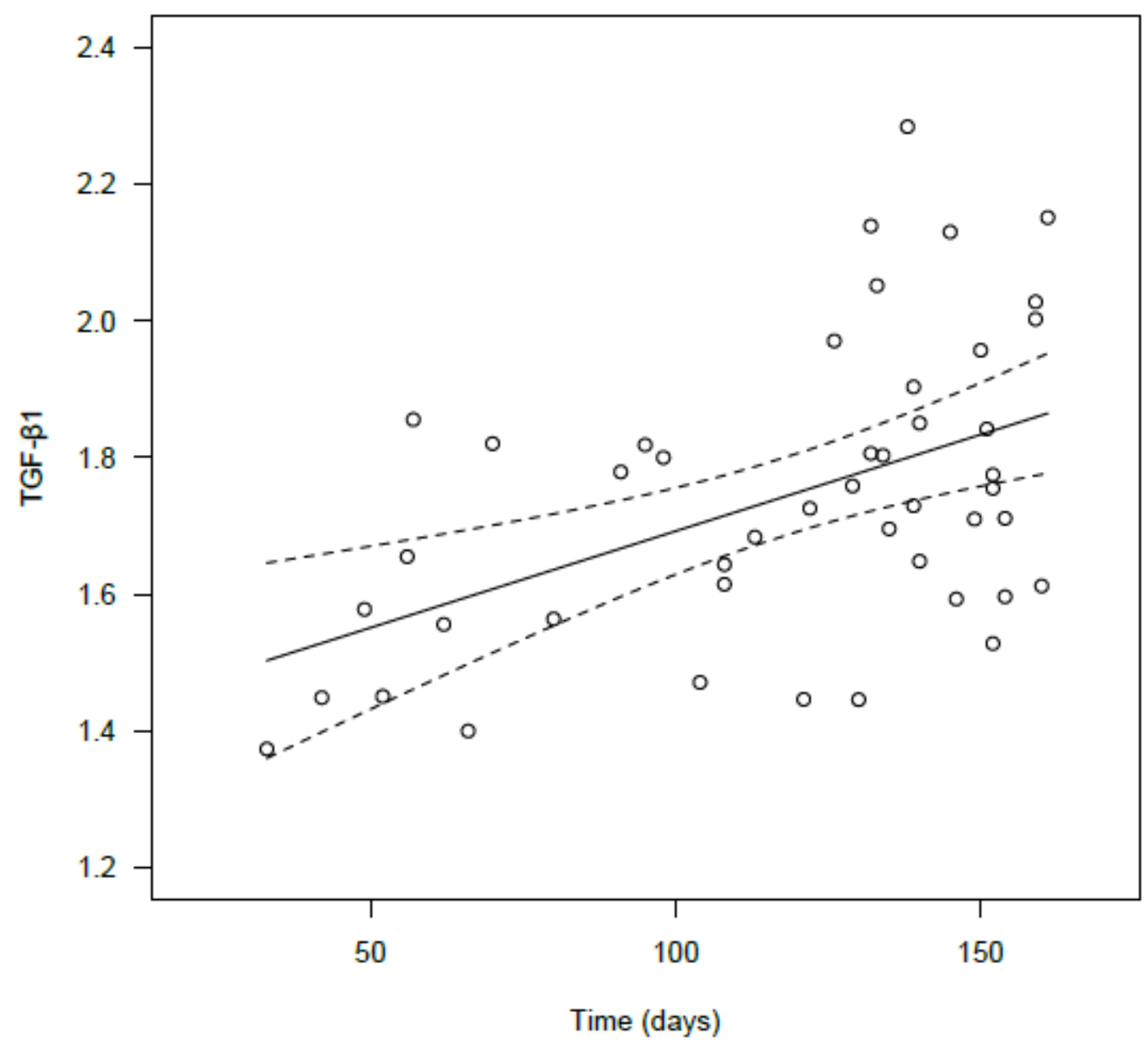


Figure 3 Ratios [95\% confidence interval] between mean values of cytokine and chemokine, in the upper airway mucosal lining fluid in healthy neonates of mothers vaccinated during pregnancy versus neonates of non-vaccinated controls.

All data is adjusted for participation in the randomized study, season of birth, maternal atopy, older siblings, and bacterial colonization.



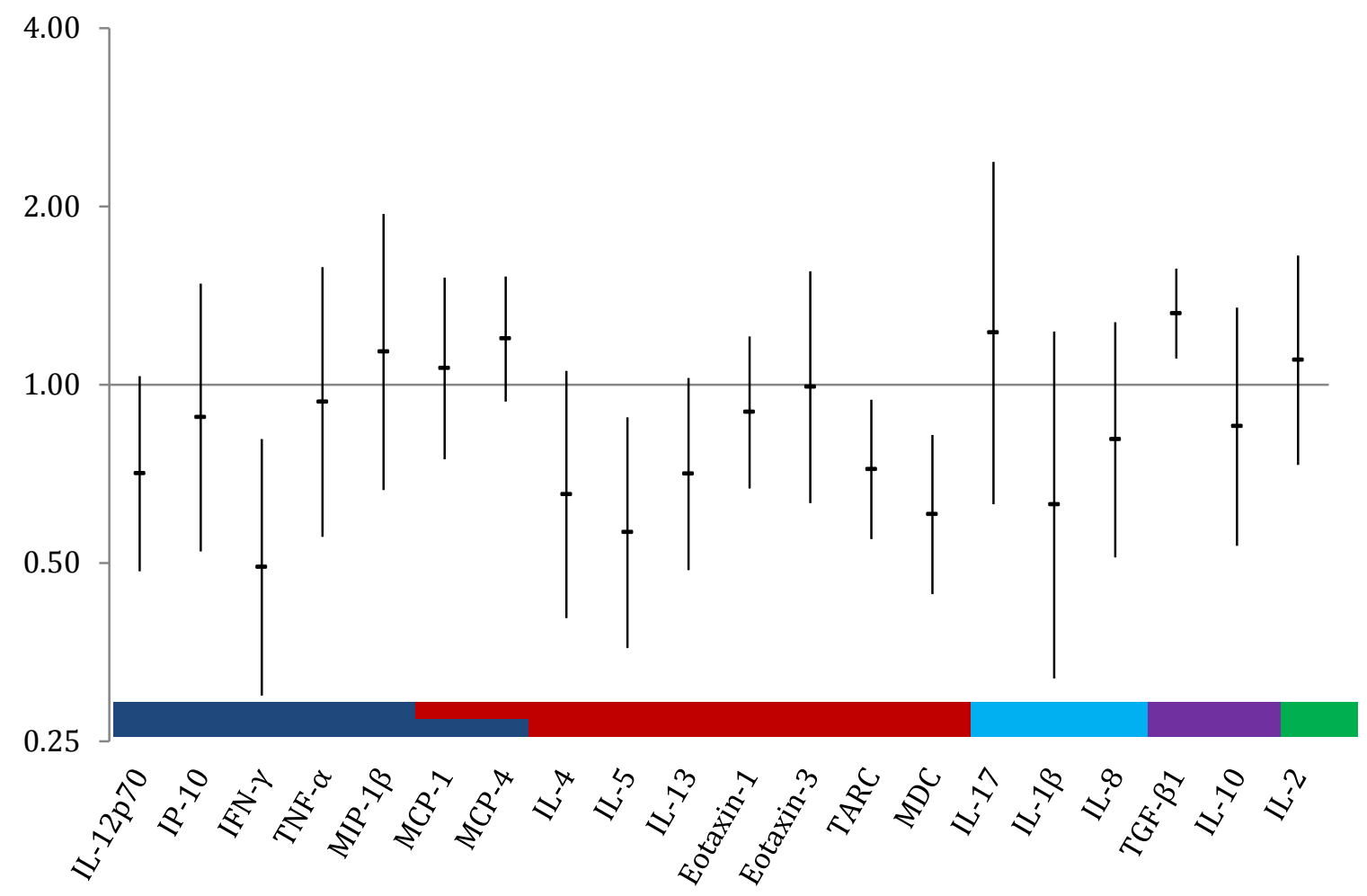




\section{Supplemental Digital Content}

\section{Airway Mucosal Immune-Suppression in Neonates of Mothers Receiving A(H1N1)pnd09 Vaccination During Pregnancy}

Anne Louise Bischoff *, MD; Nilofar V Følsgaard*, MD, PhD; Nadja H Vissing, MD; Sune Birch, MSE; Susanne Brix, MSci, PhD; Hans Bisgaard, MD, DMSci

\section{Methods}

Time from vaccination to sampling of mucosal lining fluid: The time in days from vaccination to sampling of MLF was used as continues variable in the children born to mothers vaccinated during pregnancy.

Season of birth: Date of birth was grouped into 4 seasons: winter (December-February), spring (March-May), summer (June-August) and fall (September-November).

Older siblings at birth (yes/no): Children living in the home at the time of birth were considered as siblings.

Bacterial colonization of the airway (yes/no): Aspiration from the hypopharyngeal area was done intermittently assuring no suction was applied during retraction through the oropharynx and nasopharynx. The catheter was flushed with $1 \mathrm{ml}$ of saline into a vessel for remaining secretions from the tube. Samples were transported at room temperature to the microbiology laboratories within 24 hours at the Department of Microbiological Surveillance and Research, Statens Serum Institute in Copenhagen, Denmark. Colonization with Streptococcus pneumoniae, Hemophilus influenzae and/or Moraxella catarrhalis was considered as bacterial colonization with airway pathogenic bacteria.

Maternal atopy (yes/no): Maternal atopic status was based on the history of doctor diagnosed asthma, hay fever and/or atopic dermatitis independent of sensitization. 
Exposures during $3^{\text {rd }}$ trimester of pregnancy included smoking (yes/no), alcohol consumption (yes/no), and antibiotic usage (yes/no).

Paternal atopy was defined from a history of doctor diagnosed asthma, hay fever and/or atopic dermatitis. (yes/no).

Birth details comprised way of delivery (normal birth, planned or acute sectio); birth weight; gestational age; and Apgar score below or above four one minute after birth.

Baseline antibody titers: Serum was sampled in the mothers prior to vaccination (baseline). Serum plasma levels of specific H1N1pnd09 antibodies were determined in twofold dilutions in a conventional hemagglutination-inhibition assay ${ }^{39}$. Geometric mean titers were computed from the $\log _{10}$-transformed mean.

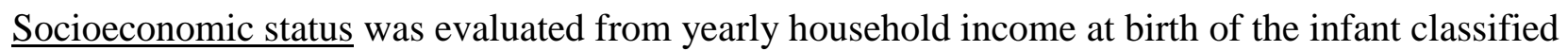
into five groups as $<54 \cdot 000 € ; 54 \cdot 000-81 \cdot 000 € ; 81 \cdot 000-108 \cdot 000 € ; 108 \cdot 000-135 \cdot 000 €$; and $>135 \cdot 000 €$. 


\section{Online Tables}

Table E1: Baseline characteristic of the study subjects

\begin{tabular}{|c|c|c|c|c|}
\hline & $\begin{array}{c}\text { Vaccinated } \\
\text { during pregnancy }\end{array}$ & $\begin{array}{c}\text { Vaccinated after } \\
\text { birth }\end{array}$ & $\begin{array}{c}\text { Non-vaccinated } \\
\text { Controls }\end{array}$ & P-value \\
\hline $\mathrm{N}$ & 51 & 105 & 332 & \\
\hline Sex (Male) \%(n) & $57(29)$ & $53(56)$ & $49(163)$ & $0.52^{\mathrm{F}}$ \\
\hline Birth weight, median(IQR) & $3 \cdot 6(3 \cdot 3-3 \cdot 9)$ & $3 \cdot 6(3 \cdot 1-3 \cdot 8)$ & $3 \cdot 5(3 \cdot 2-4 \cdot 0)$ & $0 \cdot 18^{\mathrm{A}}$ \\
\hline Gestational age, median(IQR) & $40 \cdot 0(39 \cdot 4-40 \cdot 9)$ & $40 \cdot 0(39 \cdot 1-41 \cdot 0)$ & $40 \cdot 1(39 \cdot 0-41 \cdot 1)$ & $0.83^{\mathrm{W}}$ \\
\hline Apgar $(1 \min ) \leq 4 \%(\mathrm{n})$ & 0 & $2(2)$ & $2(8)$ & $0 \cdot 88^{\mathrm{F}}$ \\
\hline Mode of delivery & & & & $0.73^{\mathrm{F}}$ \\
\hline Normal & $80(41)$ & 73(77) & $78(259)$ & \\
\hline Planned sectio & $8(4)$ & $11(12)$ & $11(37)$ & \\
\hline Acute sectio & $12(6)$ & $15(16)$ & $11(36)$ & \\
\hline Season of birth & & & & $3 \cdot 2 * 10^{-24, F}$ \\
\hline Spring & $14(7)$ & $10(11)$ & $20(65)$ & \\
\hline Summer & $12(6)$ & $59(62)$ & $21(69)$ & \\
\hline Fall & $29(15)$ & $30(32)$ & $20(66)$ & \\
\hline Winter & $45(23)$ & 0 & $40(132)$ & \\
\hline Airway bacteria* (4 week)\%(n) & $28(14)$ & $21(22)$ & $32(106)$ & $0 \cdot 09^{\mathrm{F}}$ \\
\hline Older siblings \%(n) & $51(26)$ & $65(68)$ & $61(201)$ & $0 \cdot 26^{\mathrm{F}}$ \\
\hline Baseline antibody titer, GMT(CI) & $11(8-15)$ & $13(11-17)$ & - & $0 \cdot 29^{\mathrm{A}}$ \\
\hline Antibiotics $\left(3^{\text {rd }}\right.$ trim $) \%(n)$ & $24(12)$ & $16(17)$ & $21(69)$ & $0 \cdot 47^{\mathrm{F}}$ \\
\hline Smoking $\left(3^{\text {rd }}\right.$ trim $) \%(\mathrm{n})$ & $8(4)$ & $4(4)$ & $4(13)$ & $0 \cdot 44^{\mathrm{F}}$ \\
\hline Alcohol $\left(3^{\text {rd }}\right.$ trim) $\%(\mathrm{n})$ & $6(3)$ & $7(7)$ & $3(11)$ & $0 \cdot 23^{\mathrm{F}}$ \\
\hline Income (pr. year) \%(n) & & & & $0.57^{\mathrm{F}}$ \\
\hline$<54.000 €$ & $8(4)$ & $13(14)$ & $9(29)$ & \\
\hline $54 \cdot 000-134 \cdot 000 €$ & $80(41)$ & $79(83)$ & $80(263)$ & \\
\hline$>134.000 €$ & $12(6)$ & $8(8)$ & 11(37) & \\
\hline Mothers age, median(IQR) & $32 \cdot 5(29 \cdot 5-35 \cdot 5)$ & $32 \cdot 0(29 \cdot 4-35 \cdot 6)$ & $31 \cdot 7(29 \cdot 0-35 \cdot 0)$ & $0 \cdot 45^{\mathrm{A}}$ \\
\hline Maternal atopy $\%(n)$ & $39(20)$ & $48(50)$ & $55(181)$ & $\mathbf{0} \cdot 07^{\mathrm{F}}$ \\
\hline Paternal atopy $\%(\mathrm{n})$ & $38(18)$ & $46(47)$ & $46(147)$ & $0 \cdot 52^{\mathrm{F}}$ \\
\hline $\begin{array}{l}\text { IQR: Inter Quartile Range } \\
\text { GMT: Geometric Mean Titer } \\
{ }^{\mathrm{F}} \text { Fisher's exact test } \\
{ }^{\mathrm{W}} \text { Wilcoxon Rank sum test } \\
{ }^{\mathrm{A}} \text { Anova } \\
{ }^{*} \text { Airway colonization with at }\end{array}$ & & & & \\
\hline
\end{tabular}


Table E2: Univariate analysis of association between mucosal lining fluid mediator levels and time in days from H1N1pnd09-vaccination with to sampling of mucosal lining fluid.

*Adjusted for season of birth, maternal atopy, older siblings, and bacterial colonization.

' False discovery rate $<0.05$ (Benjamini-Hochberg procedure for multiple testing correction)

$\bullet$ Th-1 • Th-2 • Th17 • Treg • Lymphocyte expansion

\begin{tabular}{|c|c|c|}
\hline Mediator & $\begin{array}{c}\text { Unadjusted } \\
{[\mathrm{CI}-\text { low, CI-high }] P \text {-value }}\end{array}$ & \begin{tabular}{l}
\multicolumn{1}{c}{ Adjusted } \\
$*[\mathrm{CI}-$ low, CI-high $] P-$ \\
value
\end{tabular} \\
\hline IL-12p70 & {$[0.98,1.00] P=0.07$} & {$[0.98,1.00] P=0.16$} \\
\hline IP-10 & {$[0 \cdot 98,1 \cdot 00] \boldsymbol{P}=\mathbf{0} \cdot \mathbf{0 3}$} & {$[0.97,1.00] P=0.08$} \\
\hline IFN- $\gamma$ & {$[0.98,1.00] P=0.05$} & {$[0.98,1.00] P=0 \cdot 17$} \\
\hline TNF- $\alpha$ & {$[0.98,1.00] P=0.15$} & {$[0.98,1.01] P=0.37$} \\
\hline MIP-1 $\beta$ & {$[0.97,1.00] P=0.07$} & {$[0.98,1.01] P=0.36$} \\
\hline MCP-1 & {$[0.99,1.00] P=0.24$} & {$[0.99,1.01] P=0.84$} \\
\hline МСР-4 & {$[1.00,1 \cdot 01] P=0.72$} & {$[1.00,1.01] P=0.58$} \\
\hline IL-4 & {$[0.99,1.01] P=0.63$} & {$[0.99,1.01] P=0.89$} \\
\hline IL-5 & {$[0.97,0.99] \boldsymbol{P}=\mathbf{0} \cdot 001$, } & {$[0.97,1.00] P=0.01$} \\
\hline IL-13 & {$[0.99,1.00] P=0.18$} & {$[0.98,1.00] P=0.09$} \\
\hline Eotaxin-1 & {$[0.99,1.00] P=0 \cdot 10$} & {$[0.99,1.01] P=0.64$} \\
\hline Eotaxin-3 & {$[0.98,1 \cdot 00] \boldsymbol{P}=\mathbf{0} \cdot 01$,} & {$[0.98,0.99] \boldsymbol{P}=\mathbf{0} \cdot \mathbf{0 0 3}$,} \\
\hline TARC & {$[0.99,1.00] \boldsymbol{P}=\mathbf{0} \cdot \mathbf{0 3}$} & {$[0.99,1.00] P=0.05$} \\
\hline MDC & {$[0.98,1.00] \boldsymbol{P}=\mathbf{0} \cdot 01$,} & {$[0.98,1.00] \boldsymbol{P}=\mathbf{0} \cdot \mathbf{0 2}$} \\
\hline IL-17 & {$[0.98,1.01] P=0.25$} & {$[0.98,1.01] P=0.62$} \\
\hline IL-1及 & {$[0.98,1.01] P=0.21$} & {$[0.98,1.01] P=0.67$} \\
\hline IL-8 & {$[0.98,1.00] \boldsymbol{P}=\mathbf{0} \cdot 03$} & {$[0.98,1.01] P=0.35$} \\
\hline TGF- $\beta 1$ & {$[1.00,1 \cdot 01] P=0 \cdot 0004$,} & {$[1 \cdot 00,1 \cdot 01] P=0 \cdot 0003$,} \\
\hline IL-10 & {$[0.98,1.00] P=0.03$} & {$[0.97,1 \cdot 00] P=0 \cdot 01$} \\
\hline IL-2 & {$[0.99,1.00] P=0.30$} & {$[0.99,1.01] P=0.76$} \\
\hline
\end{tabular}




\section{Online Figures}

Figure E1: Flowchart of study participants

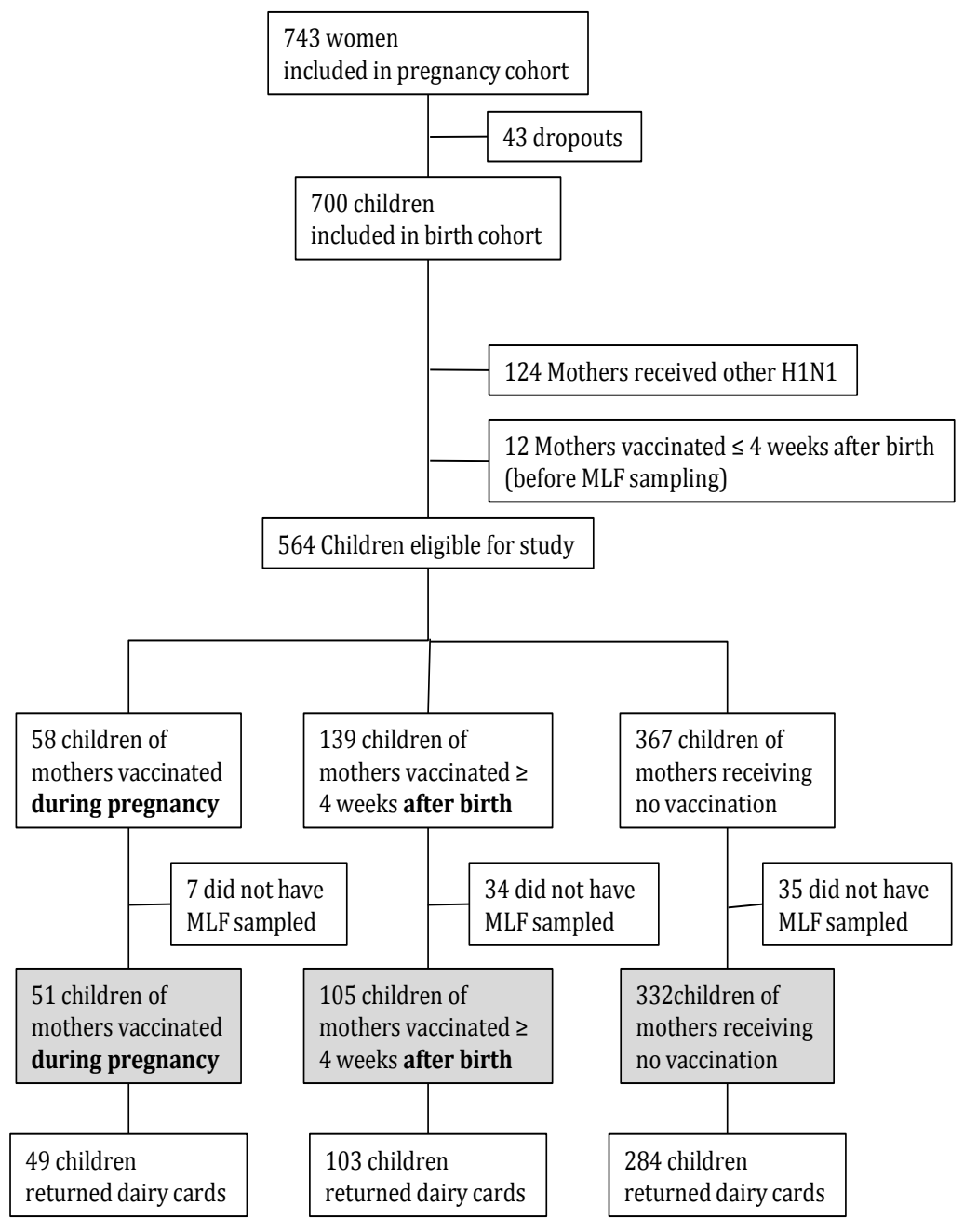


Figure E2: IL-5 concentration vs. days from H1N1pnd09-vaccination during pregnancy to sampling of mucosal lining fluid from the neonatal offspring.

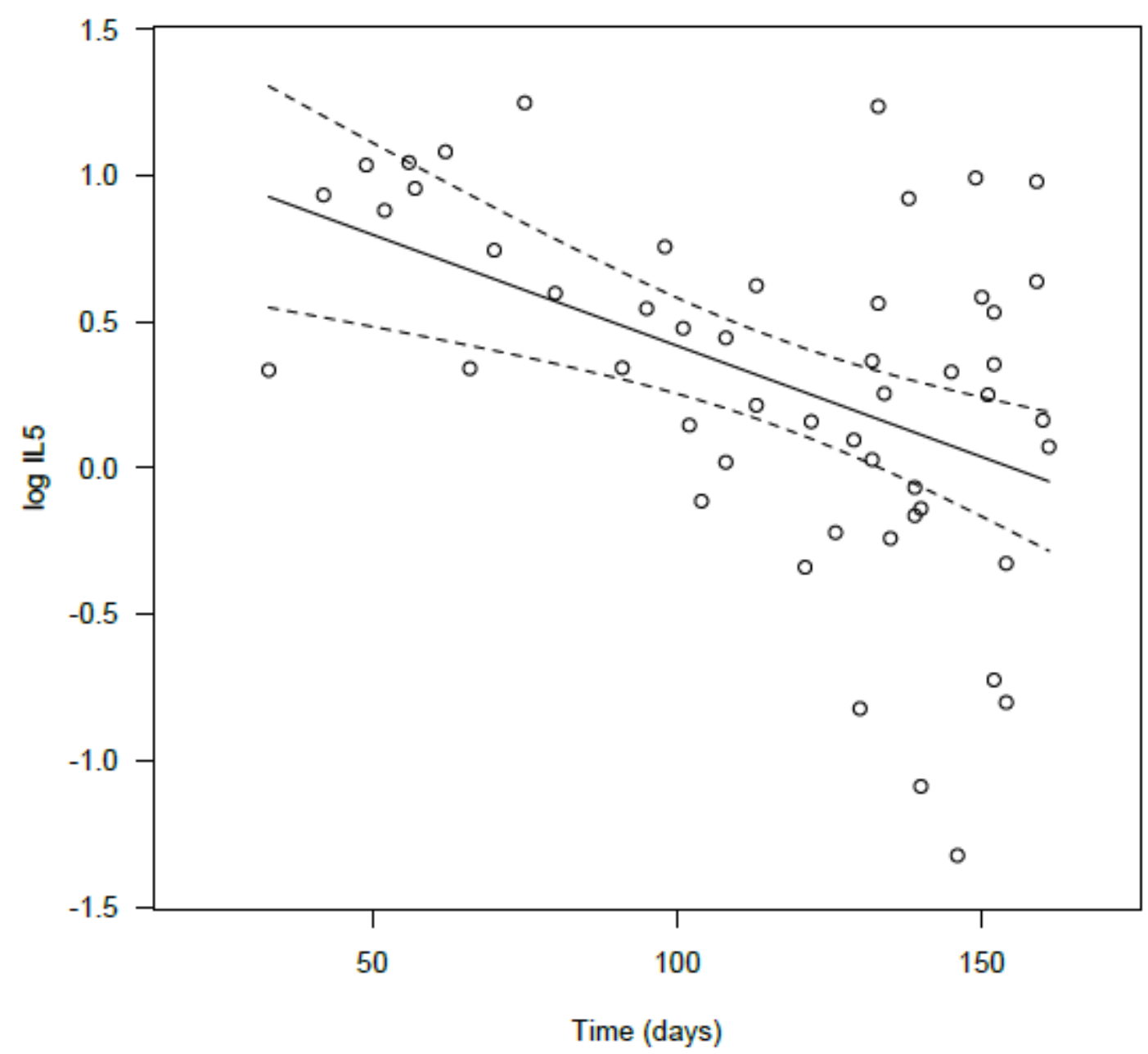

